\title{
Novel M1/M2 Heterostructure in Mo-V-M-Ta (M = Te or Sb) Complex Oxide Catalyst Revealed by Aberration Corrected HAADF STEM
}

\author{
Qian $\mathrm{He}^{1}$, Jungwon $\mathrm{Woo}^{2}$, Vadim V. Guliants ${ }^{2}$, Albina Borisevich ${ }^{1}$ \\ ${ }^{1}$ Oak Ridge National Laboratory, Oak Ridge, Tennessee 37831, USA \\ 2 School of Energy, Environment, Biological and Medical Engineering, University of Cincinnati, \\ Cincinnati, OH 45221-0012, USA
}

HAADF imaging is directly interpretable and atomic number $(Z)$ sensitive, allowing researchers to obtain structural and chemical information of the material at the atomic level with sub- $\AA$ resolution ${ }^{[1]}$. For Mo-V-M-Ta $(\mathrm{M}=\mathrm{Te}$ or $\mathrm{Sb})$ complex oxides catalysts, HAADF has been applied to reveal local cation distribution $^{[2]}$, structure and chemistry of the crystal defects ${ }^{[3]}$, polar domain structure ${ }^{[4]}$, surface $^{[5]}$ and other features in order to understand their uniquely high performance in propane (amm)oxidation reactions ${ }^{[6]}$. Previous studies have shown that the M1 phase in the Mo-V-M-Ta complex oxides (Fig 1a, note characteristic pentagon units) is mainly responsible for high activity, while another important phase, namely M2 (Fig 1b, note characteristic hexagon units), has a distinctive synergistic effect with the M1 phase by improving the selectivity of the catalytic reaction. Aiming to understand and further exploit this synergistic effect, we studied a series of M2 phase catalysts by HAADF imaging using aberration corrected STEM.

Our studies have demonstrated that M2 phase in Mo-V-Te-Ta system, unlike other previously reported M2 phases $^{[4]}$, has unusual microstructure: the side planes of the M2 phase crystals are decorated with pentagons (e.g. $\mathrm{Mo}_{6} \mathrm{O}_{21}$ ), which are building blocks for $\mathrm{M} 1$ phase (Figure 2). These pentagons appear to contain heavy cations (e.g. Ta) in higher concentration than the bulk of the material, showing brighter HAADF contrast in the center. Two types of the interface between the pentagon units and the hexagon units in the M2 phase matrix are identified. Interestingly, monolayer pentagon coverage is dominantly found associated with the type I interface (Figs. 2b,c), where multilayer coverage can always be found with the type II interface (Figs. 2d,e). In a M2 phase in a related system Mo-V-Sb-Ta, surface pentagon layers were also observed. In some areas, surface pentagon layers serve as seeds for the M1 phase attached to M2 surface. To our best knowledge, this is the first example of the M1/M2 intergrowth in Mo-V-M-Ta complex oxides catalyst.

Our microstructural observations suggest that the pentagon layers likely come from a self-assembly process of the preformed pentagon units during the synthesis, as reported by Sadakane et al. ${ }^{[7]}$, rather than post-forming surface reconstruction. The presence of $\mathrm{Ta}$, a sub-group $\mathrm{V}$ element, is found crucial for the establishment of decorated surfaces and intergrowths by comparing samples with and without Ta in composition. This clearly suggests that Ta has a stabilizing effect on the pentagon units during M2 phase formation, slowing down the precipitation and facilitating the resultant surface segregation of Ta. Correlation of the observed microstructure with the catalytic performance will also be discussed. We believe that this work will pave the way for development of novel catalysts with M1/M2 heterostructures that can improve the catalytic properties by maximizing the synergistic effect.

[1] S. J. Pennycook et al., Philos TR Soc A 367 (2009).

[2] J. J. Yu et al., Catal Commun 29 (2012).

[3] W. D. Pyrz et al., Chemistry of Materials 22 (2010).

[4] Y. H. Zhu et al., Chemistry of Materials 24 (2012).

[5] Y. Zhu et al., Angewandte Chemie 51 (2012).

[6] N. R. Shiju, and V. V. Guliants, Applied Catalysis a-General 356 (2009). 
[7] M. Sadakane et al., Angewandte Chemie 48 (2009).

[8] Research supported by the Materials Science and Engineering Division, U.S. Department of Energy (DOE), through a user project supported by ORNL's Center for Nanophase Materials Sciences, sponsored by the Scientific User Facilities Division, Office of Basic Energy Sciences, U.S. DOE, and by Chemical Sciences, Geosciences and Biosciences Division, Office of Basic Energy Sciences, U.S. DOE, under Grant \#DE-FG02-04ER15604.
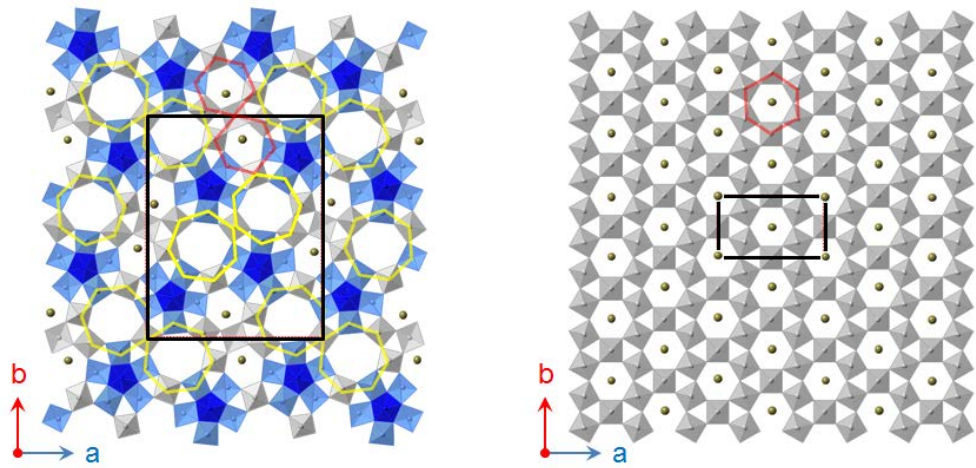

Fig 1 Polyhedral structural models of the $a b$ plane projection of (a) the M1 and (b) the M2 phases in molybdenum vanadate oxide catalysts (e.g. MoVTeTa Oxide). Pentagon units, heptagonal channels, hexagonal channels and unit cells are highlighted in blue, yellow, red and black respectively. Dilute amount of Te oxides in heptagonal channels are omitted for simplicity.

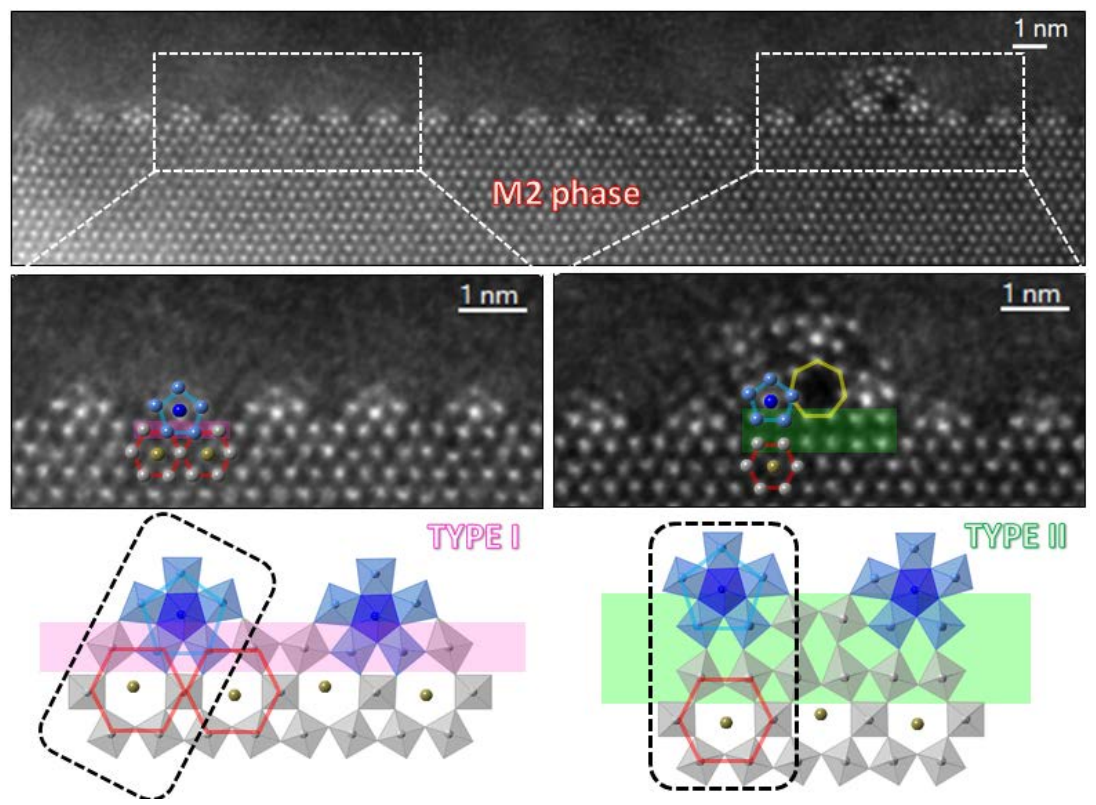

Fig 2 M1-like pentagons decorating the surface of MoVTeTa oxide M2 phase particles. (a) STEMHAADF image of the M2 phase particle surface decorated by a mono-layer of pentagon units. (b, c) and (d, e) magnified views and proposed polyhedral schematic models of Type I (purple) and Type II (green) interfaces between the M1-like pentagon units (marked in blue) and the M2 phase hexagon units (marked in red), respectively. The smallest representative units of two interfaces are highlighted in black dash lines. Notice that in the region where Type II interface is found, multiple layers of pentagons and a heptagonal channel (marked in yellow) have developed, indicative of fully formed M1 phase (Fig. 1a). 